\title{
Study on a Novel STATCOM with LC Series Filter
}

\author{
Xinwen Zhang ${ }^{1,2}$, Xiangqian Tong ${ }^{1}$ \\ ${ }^{1}$ Department of Automation\& Information Engineering, Xi' an University of Technology, Xi'an, 710048, China \\ ${ }^{2}$ Department of Electrical \& Information Engineering, Beifang University of Nationalities, Yinchuan, 750021, China
}

\begin{abstract}
In order to effectively restrain the high-frequency harmonic components in compensation current and reduce the reactive power compensation claim to the dc voltage amplitude, we present a new topology of Static synchronous compensator (STATCOM). This method changes the property of impedance by connecting capacitor in ac branch. Then, the evolution relation between new and traditional topologies of STATCOM is analyzed by the theory of circuit, and the decoupling mathematical model is deduced. Its dynamic and steady performance indexes, such as the dc voltage amplitude and total harmonic distortion of current $\left(\mathrm{THD}_{i}\right)$, are better than traditional topology. Finally, the simulation results correspond well with the theoretical analysis.
\end{abstract}

Keywords-STATCOM; series filter; decoupling control; dual circuits.

\section{INTRODUCTION}

Being its faster response, wider adjustable range and relative small size, Static synchronous compensator (STATCOM) have been applied to regulate bus voltage and to provide power factor control in distribution system in recent years [1]. The harmonic current produced by STATCOM based on inverting circuit and pulse modulation technique (PWM) injects into system. In order to improve better wave quality of compensator current, there are two main ways to be adopted. One is to change the ac filter configuration, such as the LC filter [2][3] and LCL [4][5], the other is increasing the ac filter inductance. For the latter, it not only increases the cost, but also raises the reactive power compensation demand for the dc voltage amplitude. In addition, there is a new control method that the dc voltage in STATCOM dynamically be changed according to variation of reactive power proposed [6]. However, those above measures don't reduce the demand of the dc voltage amplitude.

For the STATCOM having same capacity, the lower dc voltage, the lower cost and the lower demand for the dc voltage amplitude, and it is possible to be used in high voltage field. Moreover, it may reduce harmonic components of compensation voltage for the serial power quality controller which connected with parallel power quality by sharing dc link [7]. Based on traditional topology of STATCOM, the new topology in which the ac capacitor connected in series with ac filter inductor is proposed. Because the property of impedance of ac filter branch is changed, the fundamental component of output voltage is always lower than the source voltage when the load is inductive. Thus, the demand for the dc voltage amplitude may be reduced and the total harmonic distortion of current $\left(\mathrm{THD}_{i}\right)$ gets small. The relation between new and traditional topology of STATCOM is analyzed by duality principles of electrical circuits, and the corresponding control method is deduced. Finally, the simulation model using PSIM is established and its simulation results correspond well with the theoretical analysis.

\section{TOPOLOGY OF STATCOM}

The STATCOM compensated distribution system is shown in fig. 1 . We denote the source voltage by $u_{\mathrm{s}}$ and the fundamental component of voltage source converter (VSC) by $u_{\mathrm{c}}$.

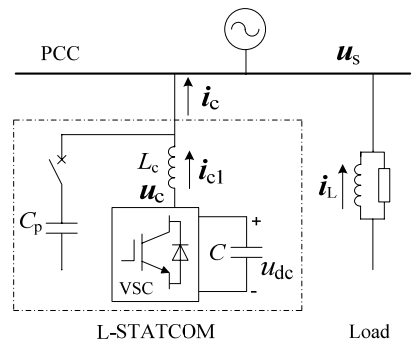

(a)

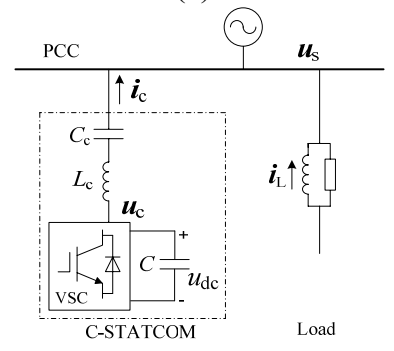

(b)

Figure 1. The reactive power compensation system based on STATCOM. (a) L-STATCOM. (b) C-STATCOM

As shown in fig.1 (a), the hybrid reactive power compensated distribution system abbreviated LSTATCOM composed of by traditional STATCOM and a parallel capacitor $C_{\mathrm{p}}$. In order to realize bidirectional reactive power compensation and in view of one-way reactive power demand of load, both of them compensate $50 \%$ reactive power. The new topology of STATCOM, abbreviated C-STATCOM shown in fig. 1 (b), is characterized in that capacitor $C_{\mathrm{c}}$ is connected in series with ac filter inductor $L_{\mathrm{c}}$. Obliviously, there is dual relation between L-STATCOM and C-STATCOM. 
It is assumed that SPWM modulation method is used to VSC, and $\lambda$ represents the modulation index. According to the principle of PWM modulation, the ac/dc voltage relationship can be written as:

$$
U_{\mathrm{c}}=\frac{k_{\mathrm{c}} \lambda u_{\mathrm{dc}}}{\sqrt{2}}
$$

Where $k_{\mathrm{c}}=1 / 2 . U_{\mathrm{c}}$ in fig. 1 (a) is bigger than $U_{\mathrm{s}}$, where $\lambda \in[0,1], \quad U_{\mathrm{c}}$ in fig. 1 (b) is smaller than $U_{\mathrm{s}}$, where $\lambda \in[-$ $1,1]$.

\section{PARAMETERS AND CHARACTERISTIC OF C- STATCOM}

\section{A. Parameter selection}

The relationship between $I_{\mathrm{c}}$ of C-STATCOM and LSTATCOM and $U_{c}$ is shown in fig.2. Line segment AB indicates the bidirectional characteristic of traditional STATCOM. Line segment EF indicates the characteristic of L-STATCOM, and CD indicates the characteristic of C-STATCOM.

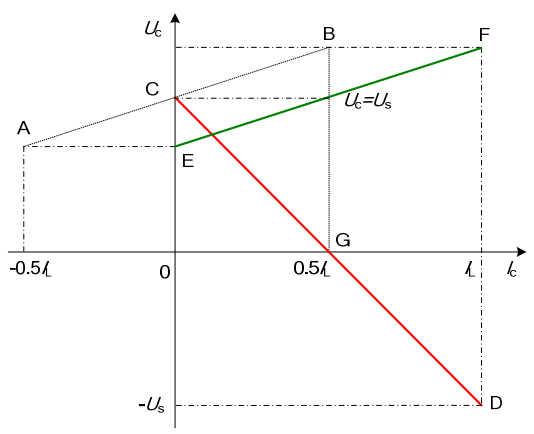

Figure 2. The relation between $I_{\mathrm{c}}$ and $U_{\mathrm{c}}$

Fig. 2 infers that the amplitude of $U_{\mathrm{c}}$ in L-STATCOM varies around $U_{s}$, and its variation depends on the reactance ratio of filter inductor. The amplitude of $U_{\mathrm{c}}$ in C-STATCOM varies from $-U_{s}$ to $U_{s}$. fig.3.

The ac equivalent circuit of VSC in fig. 1 is show in

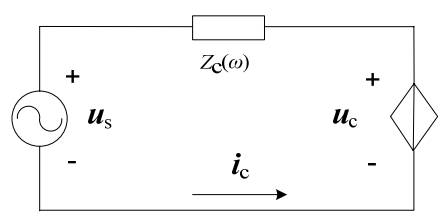

Figure 3. The ac equivalent circuit of STATCOM

In fig.3, we denote the fundamental-frequency impedance of ac filter branch as $Z_{\mathrm{c}}(\omega)$, then its corresponding equations of L-STATCOM and CSTATCOM are given as follows:

$$
\begin{gathered}
Z_{\mathrm{c} . \mathrm{L}}(\omega)=R_{\mathrm{c}}+j \omega L_{\mathrm{c}} \\
Z_{\mathrm{c} . \mathrm{C}}(\omega)=R_{\mathrm{c}}+j\left(\omega L_{\mathrm{c}}-\left(\omega C_{\mathrm{c}}\right)^{-1}\right)
\end{gathered}
$$

Where $\omega$ is angular frequency of $u_{\mathrm{s}}$, and $R_{\mathrm{c}}$ is equivalent resistance of $L_{\mathrm{c}}$ and VSC loss, and its value ranges from 5 to $10 \% \omega L_{\mathrm{c}}$. It is usually omitted in order to simplify formula derivation. $L_{\mathrm{c}}$ in $Z_{\mathrm{c}}(\omega)$ is decided by selecting principle of traditional STATCOM. The selection of $C_{\mathrm{c}}$ should satisfy $Z_{\mathrm{c} . \mathrm{C}}\left(\omega_{\mathrm{s}}\right) \approx Z_{\mathrm{c} . \mathrm{L}}\left(\omega_{\mathrm{s}}\right) \quad\left(\omega_{\mathrm{s}}\right.$ is angular frequency of carrier signal) in order to be compared with L-STATCOM.

Assuming load reactive power is $Q_{\mathrm{N}}$ and its inductive characteristic is positive. After reactive power is completely compensated, $C_{\mathrm{c}}$ can be written as:

$$
C_{\mathrm{c}}=\frac{Q_{\mathrm{N}}}{\omega\left(\omega L_{\mathrm{c}} Q_{\mathrm{N}}+3 U_{\mathrm{s}}\left(U_{\mathrm{s}}-U_{\mathrm{c}}\right)\right)}
$$

\section{B. Property analysis and control}

The property index of STATCOM can be measured primarily by total harmonic distortion of current $\left(\mathrm{THD}_{i}\right)$ and cost of main components of VSC. Therefore, the properties of C-STATCOM should be analyzed.

Combined the equation (1) and reactive power compensation mechanism of STATCOM, it can be induced that

$$
u_{\mathrm{dc}}=\frac{\sqrt{2}\left(3 U_{\mathrm{s}}^{2}+(-1)^{k}\left|Z_{\mathrm{c}}(\omega)\right| Q_{\mathrm{N}}\right)}{3 k_{\mathrm{c}} \lambda U_{\mathrm{s}}}
$$

Where $k=-1$ when $Z_{\mathrm{c}}(\omega)=Z_{\mathrm{c} . \mathrm{L}}$, and $k=1$ when $Z_{\mathrm{c}}(\omega)=Z_{\mathrm{c} . \mathrm{L}}$

According to the calculation principle of $\mathrm{THD}_{i}$, it can be written as

$$
\operatorname{THD}_{i}=\frac{3 U_{\mathrm{s}}^{2}+(-1)^{k}\left|Z_{\mathrm{c}}(\omega)\right| Q_{\mathrm{N}}}{\pi k_{\mathrm{c}} \lambda Q_{\mathrm{N}}} \sqrt{\sum_{k=1, n=1}^{\infty} \frac{J_{\mathrm{k}}^{2}\left(\frac{\lambda n \pi}{2}\right)}{n^{2} \Delta}}
$$

$n=1,3,5 \ldots, k=2 l, k \neq 6 l(l=1,2,3 \ldots), n=2,4,6 \ldots, k=2 l$ $1, k \neq 6 l-3 \quad(l=1,2,3 \ldots), J_{\mathrm{k}}(x)$ is $\mathrm{n}$ order Bessel function. When $Z_{\mathrm{c}}(\omega)=Z_{\mathrm{c} . \mathrm{L}}(\omega), \Delta$ in $\mathrm{THD}_{i}$ calculation can be given as

$$
\Delta=\left(\left(k \omega \pm n \omega_{\mathrm{s}}\right) L_{\mathrm{c}}\right)^{2}
$$

When $Z_{\mathrm{c}}(\omega)=Z_{\mathrm{c} . \mathrm{C}}(\omega), \Delta$ in $\mathrm{THD}_{i}$ calculation can be given as

$$
\Delta=\left(\left(k \omega \pm n \omega_{\mathrm{s}}\right) L_{\mathrm{c}}-\left(\left(\left(k \omega \pm n \omega_{\mathrm{s}}\right) C_{\mathrm{c}}\right)^{-1}\right)\right)^{2}
$$

Combine (5) and (6), the relationship of $\mathrm{THD}_{i}$ between L-STATCOM (without $C_{\mathrm{p}}$ ) and of C-STATCOM is

$$
\frac{\mathrm{THD}_{i . \mathrm{C}}}{\mathrm{THD}_{i . \mathrm{L}}} \approx \frac{u_{\mathrm{dc.} \mathrm{C}}}{u_{\mathrm{dc} . \mathrm{L}}}
$$

From (7), decreasing the demand of dc voltage can efficiently improve the quality of its output current in condition of same operation conditions and parameters. 
The mathematical model of traditional STATCOM is shown in reference [6]. This paper mainly discusses the mathematical model of C-STATCOM. By inspecting fig. 1(b), the voltage equilibrium of ac branch can be written as

$$
\left\{\begin{array}{c}
L_{\mathrm{c}} \frac{d \mathbf{i}_{\mathrm{c}}}{d t}+\boldsymbol{u}_{\mathrm{cs}}+\boldsymbol{u}_{\mathrm{c}}=\boldsymbol{u}_{\mathrm{s}} \\
\boldsymbol{i}_{\mathrm{c}}=C_{\mathrm{c}} \frac{d \boldsymbol{u}_{\mathrm{cs}}}{d t}
\end{array}\right.
$$

Assuming A phase voltage of $u_{\mathrm{s}}$ and $\mathrm{d}$ axis of synchronization frame as reference phase, we get the dq axis components expression of Laplace transformation corresponding to (8).

$$
\left\{\begin{array}{l}
{\left[\begin{array}{l}
U_{\text {c.d }} \\
U_{\text {c.q }}
\end{array}\right]=\left[\begin{array}{cc}
-s L_{\mathrm{c}} & \omega L_{\mathrm{c}} \\
-\omega L_{\mathrm{c}} & -s L_{\mathrm{c}}
\end{array}\right]\left[\begin{array}{l}
I_{\mathrm{c} . \mathrm{d}} \\
I_{\mathrm{c} . \mathrm{q}}
\end{array}\right]+\left[\begin{array}{c}
U_{\mathrm{s}} \\
0
\end{array}\right]-\left[\begin{array}{l}
U_{\mathrm{cs} . \mathrm{d}} \\
U_{\text {cs.q }}
\end{array}\right]} \\
{\left[\begin{array}{l}
I_{\mathrm{c} . \mathrm{d}} \\
I_{\mathrm{c} . \mathrm{q}}
\end{array}\right]=\left[\begin{array}{cc}
s C_{\mathrm{c}} & -\omega C_{\mathrm{c}} \\
\omega C_{\mathrm{c}} & s C_{\mathrm{c}}
\end{array}\right]\left[\begin{array}{l}
U_{\text {cs.d }} \\
U_{\text {cs. }}
\end{array}\right]}
\end{array}\right.
$$

This paper uses the feedforward decoupling method to deal with coupling term. The details are shown in fig.4.

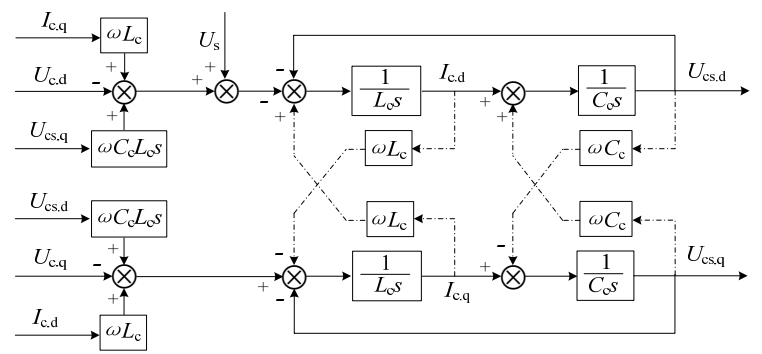

Figure 4. The decoupling model of ac branch of VSC in synchronous frame

After decoupling, dq axis coupled mathematical model in (9) is transformed to dq axis mutual independent mathematical model. Because dq axis has same control structure, taking $\mathrm{q}$ axis for example, fig.5 shows its control structure.

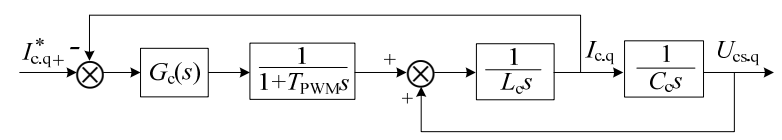

Fig.5: The control structure of reactive current

In fig. $5, I_{\text {c.q }}^{*}$ represents the reactive command current, $G_{\mathrm{c}}(s)$ represents $\mathrm{q}$ axis current controller, $\mathrm{T}_{\mathrm{PWM}}$ is approximate equivalent for PWM to control delay action.

For constant dc voltage controls applied in STATCOM, the equation of dc voltage can be written as

$$
C \frac{d u_{\mathrm{dc}}}{d t}=i_{\mathrm{dc}}
$$

$i_{\mathrm{dc}}$ is given current $I_{\mathrm{c} . \mathrm{d}}^{*}$ which is the active power component of ac current for STATCOM to maintain given $\mathrm{dc}$ voltage. Its control structure is

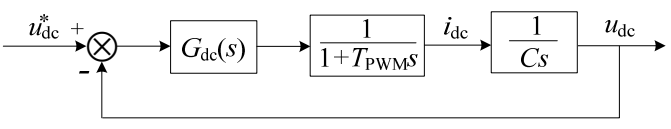

Figure 6. The control structure of dc voltage

$u_{\mathrm{dc}}^{*}$ is the given dc voltage, and $G_{\mathrm{dc}}(s)$ is dc voltage controller.

\section{Simulation RESUlts}

Assuming that the three-phase load in distribution system is balancing, the reactive power compensation system based on L-STATCOM and C-STATCOM is shown in fig.1. And the related simulation parameters with PSIM are listed in Table 1.

TABLE I. THE PARAMETERS OF STATCOM SIMULATION SYSTEM

\begin{tabular}{lll}
\hline Symbol & C-STATCOM & L-STATCOM \\
\hline $\boldsymbol{u}_{\mathrm{s}}$ & $380 \mathrm{~V} / 50 \mathrm{H}$ & $380 \mathrm{~V} / 50 \mathrm{H}$ \\
$Q_{\mathrm{N}}$ & $200 \mathrm{kVar}$ & $200 \mathrm{kVar}$ \\
$L_{\mathrm{c}}$ & $1 \mathrm{mH}$ & $1 \mathrm{mH}$ \\
$\mathrm{IGBT}$ & $1200 \mathrm{~V} / 600 \mathrm{~A}$ & $1700 \mathrm{~V} / 300 \mathrm{~A}$ \\
$C_{\mathrm{c}}$ & $1803 \mu \mathrm{F}$ & $/$ \\
$C_{\mathrm{p}}$ & $/$ & $2193 \mu \mathrm{F}$ \\
$u_{\mathrm{dc}}^{*}$ & $600 \mathrm{~V}$ & $800 \mathrm{~V}$ \\
\hline
\end{tabular}

From two aspects of starting and running, the performance of the C-STATCOM is analyzed in the following discussion. Taking one phase of $u_{\mathrm{s}}$ for example, the waveform of $\boldsymbol{i}_{\mathrm{c} 1}$ and $u_{\mathrm{dc}}$, which produced by LSTATCOM and C-STATCOM, are respectively shown in fig.7 (a) and (b). 


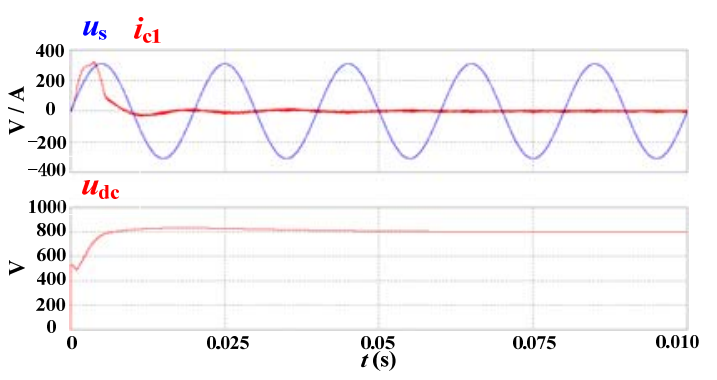

(a)

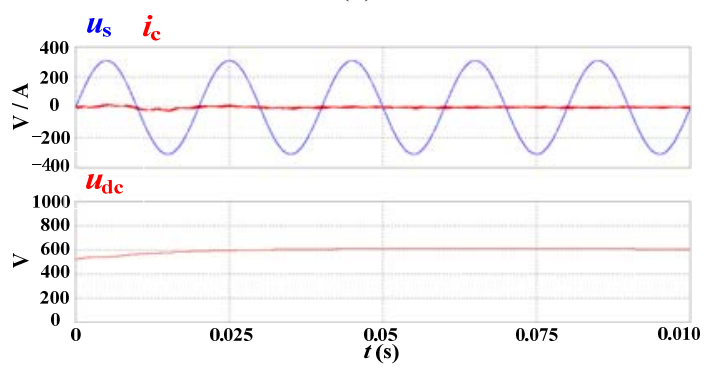

(b)

Figure 7. The waveform of $\boldsymbol{i}_{\mathrm{cl}}$ and $u_{\mathrm{dc}}$ when STATCOM starts. (a) LSTATCOM. (b) C-STATCOM

Compared (a) with (b), the peak value of $\boldsymbol{i}_{\mathrm{c} 1}$ and $u_{\mathrm{dc}}$ produced by L-STATCOM is greater than C-STATCOM. Obviously, C-STATCOM is easy to start.

When reactive load, that is $Q$, changes from $0 \sim 0.5 Q_{\mathrm{N}}$, the waveform of $\boldsymbol{i}_{\mathrm{c}}$ and $\boldsymbol{u}_{\mathrm{c}}$ produced by L-STATCOM $\left(C_{\mathrm{p}}\right.$ is offline) and C-STATCOM are respectively shown in fig. 8 (a) and (b).

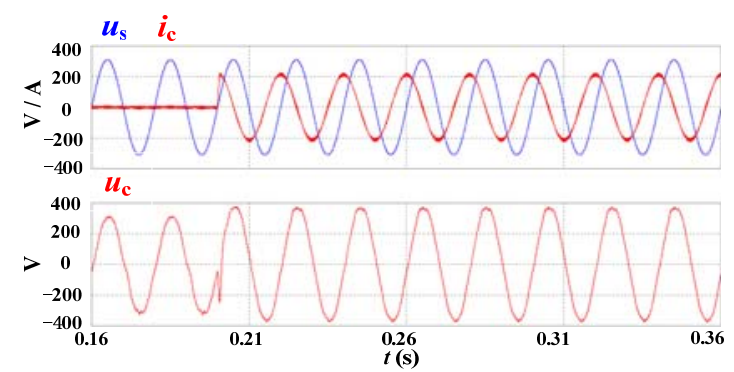

(a)

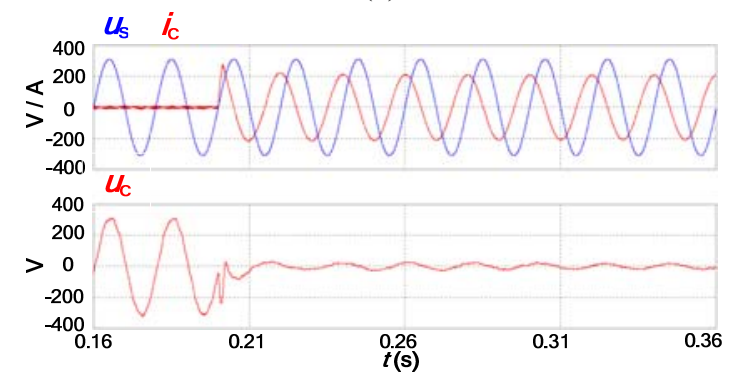

(b)

Figure 8. The wave of parameters when $Q$ from 0 to $0.5 Q_{\mathrm{N}}$. (a) LSTATCOM. (b) C-STATCOM $\boldsymbol{u}_{\mathrm{c}}$ is acquired by first order lowpass filter whose cutoff frequency is $500 \mathrm{~Hz}$. Being its inherent delay characteristics, the phase of $\boldsymbol{u}_{\mathrm{c}}$ is slightly lagging behind $\boldsymbol{u}_{\mathrm{s}}$. Compared (a) with (b), the dynamic and steady performance of L-STATCOM and C-STATCOM are basically same. $\boldsymbol{u}_{\mathrm{c}}$ produced by L-STATCOM is in phase with $\boldsymbol{u}_{\mathrm{s}}$ but its amplitude is greater than the latter, and $\boldsymbol{u}_{\mathrm{c}}$ produced by C-STATCOM is almost zero when Q equals $0.5 Q_{\mathrm{N}}$. In addition, the relation between $\mathrm{THD}_{i}$ and per-unit for $Q$ that $0.5 Q_{\mathrm{N}}$ is as a benchmark is shown in fig.9.

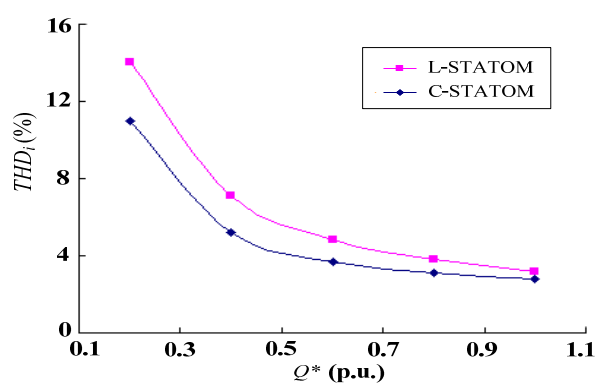

Figure 9. The comparison of $\mathrm{THD}_{i}$ between L-STATCOM without $C_{\mathrm{p}}$ and C-STATCOM

When $Q$ changes from $0.5 Q_{\mathrm{N}}$ to $Q_{\mathrm{N}}$, the waveform of $\boldsymbol{i}_{\mathrm{c}}$ and $\boldsymbol{u}_{\mathrm{c}}$ produced by L-STATCOM ( $C_{\mathrm{p}}$ is online) and CSTATCOM are respectively shown in fig.10(a) and (b).

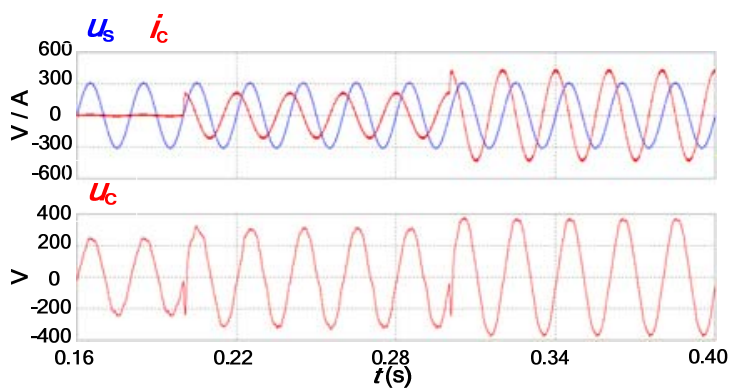

(a)

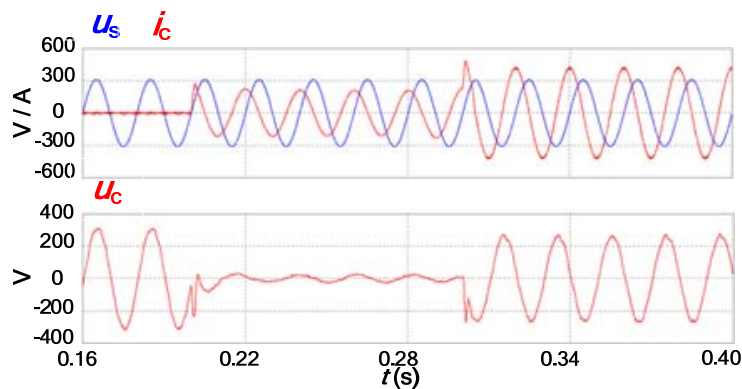

(b)

Figure 10 The waves of parameters when $Q$ from $0.5 Q_{\mathrm{N}}$ to $Q_{\mathrm{N}}$. (a) LSTATCOM. (b) C-STATCOM

Compared (a) with (b), $\boldsymbol{u}_{\mathrm{c}}$ produced by L-STATCOM is in phase with $\boldsymbol{u}_{\mathrm{s}}$ but its amplitude is much greater than the latter, and $\boldsymbol{u}_{\mathrm{c}}$ produced by C-STATCOM nearly equals $\boldsymbol{u}_{\mathrm{s}}$ but two of them are reverse in phase when Q equals $Q_{\mathrm{N}}$ 
The relation between $\mathrm{THD}_{i}$ and per-unit for $Q$ that $Q_{\mathrm{N}}$ is as a benchmark is shown in fig. 11 .

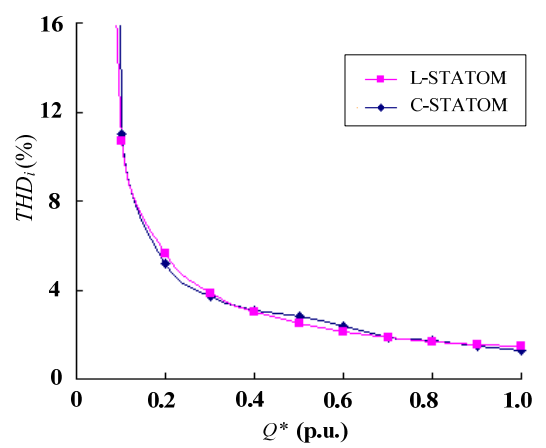

Figure 11. The comparison of $\mathrm{THD}_{i}$ between L-STATCOM and CSTATCOM

\section{CONCLUSIONS}

In the proposed topology of STATCOM, it can effectively reduce the demand for the dc voltage amplitude, which is general about $25 \%$ and makes the selected device has a greater safety margin, or made it possible to choose switching device having low voltage stress. At the same time, its performance index, such as starting current and $\mathrm{THD}_{i}$, exceed the traditional topology of STATCOM, and its $\mathrm{THD}_{i}$ is similar with hybrid reactive compensation system. Next, we will do the experiment to verify the theoretical analysis.

\section{ACKNOWLEDGEMENTS}

This research work was supported in part by the Key Discipline Special Foundation of Shaanxi Province, China, under Grant 5X1301, the Specialized Research Fund for the Doctoral Program of Higher Education, China, under Grant 20126118110009, and the Research Fund of Beifang University of Nationalities, China, under Grant 2014 XBZ05.

\section{REFERENCES}

[1] Singh.B, Saha.R, Chandra.A, et al.Static Synchronous Compensators (STATCOM): a Review[J]. IET Power Electro, 2009, 2(4):297-324.

[2] Fei Rong, An Luo, Hongbin Pan. Improvement of LC Output Filter and Its Optimization in STATCOM[J]. Electric Power Automation Equipment, 2007, 27(9):83-87.

[3] Tang Ping, Xiangen Yin, Zhe Zhang. Topology Analysis of the Output Filter in D-STATCOM and Its Parameters Multi-objective Optimal Design[C]. Proceedings of 2011 International Conference on Advanced Power System Automation and Protection(APAP)[C].2011, 998- 002.

[4] Xuefeng Hu, Wei Yi, Yihan Chen. A Control Strategy for Gridconnected Inverters with LCL Filters[J], Proceedings of the CSEE, 2012, 32(27):142-148.

[5] Cunping Wang, Xianggen Yin, Zhang Zhe. Characteristic Analysis and Parameter Design of Output LCL Filter in D-STATCOM[J]. Transactions of China Electrotechnical Society, 2011, 26(12):99-105.

[6] Xiaobing Zhang, Yanru Zhong. Optimal Dynamic Hierarchical Control of the STATCOM DC Side Voltage [J], Proceedings of the CSEE, 2009, 29(33):60-67.

[7] Karanki. K, Geddada.G, Mishra.M.K. A Modified Three-Phase FourWire UPQC Topology with Reduced DC-Link Voltage Rating[J]. IEEE Transactions on Power Electronics, 2013, 60(9):3555-3566. 